\title{
Conclusions du congrès
}

\section{Nive Voisine}

Volume 36, 1969

URI : https://id.erudit.org/iderudit/1007298ar

DOI : https://doi.org/10.7202/1007298ar

Aller au sommaire du numéro

Éditeur(s)

Les Éditions Historia Ecclesiæ Catholicæ Canadensis Inc.

ISSN

0318-6172 (imprimé)

1927-7067 (numérique)

Découvrir la revue

Citer ce document

Voisine, N. (1969). Conclusions du congrès. Sessions d'étude - Société canadienne d'histoire de l'Église catholique, 36, 93-96. https://doi.org/10.7202/1007298ar

Tous droits réservés @ Les Éditions Historia Ecclesiæ Catholicæ Canadensis Inc., 1970
Ce document est protégé par la loi sur le droit d'auteur. L'utilisation des services d'Érudit (y compris la reproduction) est assujettie à sa politique d'utilisation que vous pouvez consulter en ligne.

https://apropos.erudit.org/fr/usagers/politique-dutilisation/ 


\section{Conclusions du congrès}

En 1967, à l'occasion d'un colloque tenu à l'Institut d'histoire de l'Université Laval, Pierre Savard rappelait avec justesse que \& l'histoire du clergé canadien-français qui fut si étroitement mêlé au destin de notre peuple reste à écrire ». On a, soulignait-il, étudié son attitude politique, son œuvre dans l'éducation, ses préoccupations sociales, mais d'immenses zones n'ont pas été explorées, par exemple - et je cite toujours Pierre Savard - « la vie interne du clergé, son recrutement, sa formation, sa spiritualité, la physionomie et l'action des diverses familles religieuses, la discipline ecclésiastique, la pensée ecclésiologique, les relations entre les clercs et leurs supérieurs ${ }^{1}{ }^{~}$. Et surtout il manque une étude d'ensemble sur le clergé canadien-français.

Il faut rappeler cependant que la Société canadienne d'Histoire de l'Église catholique apporte, depuis plusieurs années, une contribution remarquable à l'étude de l'histoire du clergé. Et elle continue aujourd'hui en demandant aux conférenciers de faire connaître certaines communautés religieuses du XIX siècle. C'est un apport intéressant à l'étude de la physionomie et de l'action de quelques familles religieuses.

Pour terminer cette journée d'étude, je ne vais pas vous résumer les conférences, mais simplement vous faire part des réflexions qu'elles ont suscitées en moi.

L'histoire des communautés religieuses ne pouvait être mieux introduite que par le texte de M. Claude Galarneau, Les communautés religieuses, 1837-1914. L'inventaire qu'il nous a livré est à la fois neuf et intéressant. Ces statistiques, même si elles ne sont pas définitives, révèlent non seulement une floraison surprenante de communautés religieuses, mais un lien très étroit entre l'implantation de ces familles religieuses et les événements politiques. Ce qui nous montre, et la conférence en a été un exemple, tout ce qu'on peut tirer des statistiques pourvu qu'on les dépasse. Il faut également savoir gré à $M$. Galarneau d'avoir souligné qu'il reste plusieurs problèmes ou points de recherche à explorer; nous faisons nôtre sa demande de solides monographies sur l'histoire des communautés canadiennes et françaises.

Il était difficile, dans l'œuvre immense des Oblats de Marie-Immaculée, de choisir ce qui les caractérisait davantage. Le Père Gabriel

1 Pierre Savard, « La vie du clergé québecois au XIX` siècle •, Recherches sociographiques, VIII, 3 (sept.-déc. 1967), p. 259 et suiv. 
Bernier a fait preuve de flair et de science en parlant surtout de l'évangélisation des pauvres. C'est avec raison qu'il a souligné l'importance des missions (ou retraites) paroissiales et des campagnes contre l'intempérance. C'est une étude qu'il faudra poursuivre même s'il en est un peu question dans la monumentale Histoire documentaire de la Congrégation des Missionnaires Oblats de Marie-Immaculée dans l'Est du Canada, du père Gaston Carrière. Il faudrait surtout qu'on fasse une histoire de la prénication à travers l'histoire de certaines communautés ou de certains prédicateurs; elle est nécessaire pour tracer le portrait du Canada français du XIX ${ }^{e}$ siècle. Il faut que les communautés nous aident à éclairer certains problèmes : la spiritualité sous-jacente à la prédication, les modèles étudiés, la formation théologique des prédicateurs, les effets connus ou cachés, etc. Déjà, les journaux de l'époque nous rapportent des récits des missions paroissiales, mais dans quel style! Voyez par vous-mêmes :

Si les échos pouvaient répéter les secrets sentiments du cœur, bien au loin retentiraient ces mots d'actions de grâces : merci mon Dieu !... Merci pour les bienfaits signalés que vous venez de déverser sur la belle paroisse de St-Grégoire le Grand. Merci, pour les bénédictions abondantes que votre main libérale a fait pleuvoir sur les heureux habitants de cette paroisse privilégiée. Merci, enfin, pour avoir envoyé vers nous, un de ces hommes vraiment apostoliques, dont la noble tâche est de ramener au bercail du Bon Pasteur, les brebis infidèles qui s'en étaient éloignées.

Lundi, le son des cloches annonçait le départ du Révérend Père. Accompagné du digne Pasteur de la paroisse et de son zélé Collaborateur, il se dirigea vers la station. 50 voitures, portant des pavillons, lui faisaient cortège; on voulait une dernière fois, témoigner sa reconnaissance à celui qui venait de faire à tous, un si grand bien. Les chemins avaient été préparés avec soin, on voyait des arcs de triomphe s'élever sur le passage de l'homme de Dieu ${ }^{2}$.

Nous aimerions connaître la version du prédicateur !

M. François De Lagrave nous a présenté les Frères des Écoles chrétiennes dans la moitié du XIX ${ }^{\bullet}$ siècle. Son travail nous met déjà sur la piste de deux recherches. Il faut étudier le rôle pédagogique important des communautés françaises : les manuels qu'elles ont produits, mais aussi les méthodes d'enseignement et leur rôle sur la langue parlée. De même faut-il connaître le plus possible de leur idéologie. La plupart des sujets venus au Québec étaient ultramontains, mais il faudrait quand même examiner leur formation, l'influence qu'ils ont subie en France, les auteurs qu'ils ont lus, etc. Nous aurions ainsi des vues nouvelles sur l'impact des problèmes français sur le Canada du XIX ${ }^{\bullet}$ siècle.

Sœur Liliane Héroux, de l'Institut des Sœurs de la Charité de Québec, nous a fait connaître un bel exemple du lien étroit entre

2 Le Journal des Trois-Rivières, février 1875. 
l'histoire d'une communauté et celle d'un milieu (la ville de Québec). Cet exemple est d'autant plus intéressant que cette famille religieuse s'adapte régulièrement aux besoins des groupes et des personnes, passant des ouvres sociales à l'éducation et, d'une certaine façon, au travail missionnaire. Il y a beaucoup à tirer de cette histoire des Sours de la Charité de Québec qui est d'ailleurs en grande partie écrite.

En se penchant sur le Bon-Pasteur de Québec, Sœur Thérèse Boucher nous a révélé une fondation québécoise (au sens le plus précis du terme) qui s'est donné, elle aussi, des buts très clairs répondant aux besoins de l'époque. Il faudrait étudier, d'une façon plus complète, l'action des grandes personnalités (le Père Saché, le grand-vicaire Cazeau, l'abbé A.-A. Blais) sur cette communauté. Nous aurions un autre chapitre intéressant de l'histoire de la spiritualité canadienne.

Enfin, les conférenciers de ce soir nous ont présenté deux des plus anciennes communautés du pays : les Jésuites et les Ursulines. Des premiers, dont le Père Georges-Émile Giguère nous a décrit le retour, nous dirons que l'histoire religieuse du $\mathrm{XIX}^{\bullet}$ siècle sera incomplète tant qu'on n'aura pas une étude de l'action de la Compagnie de Jésus dans tous les diocèses et dans tous les domaines. Ce sera une œuvre immense, mais qui va ouvrir des perspectives intéressantes sur les problèmes religieux de l'époque. Quant à Sœur Emmanuel Chabot, elle nous a prouvé que le renouveau de sa communauté au XIX ${ }^{\bullet}$ siècle est venu de religieuses étrangères, anglaises ou américaines, qui ont apporté une tradition culturelle très riche et qui ont donné un bel exemple de l'entente " raciale ».

Pour terminer, je ne veux que vous livrer quelques considérations générales très brèves.

L'essor des communautés religieuses au Canada coïncide avec le mouvement général de renouveau de l'Église universelle. Le dynamisme dont fait preuve l'Église canadienne se retrouve ailleurs et s'alimente souvent aux sources européennes. Ici comme partout à la fin du XIX ${ }^{\bullet}$ siècle, la vie religieuse prend une physionomie nouvelle, plus contemporaine : augmentation considérable de l'effectif total, favorisée par la multiplication des congrégations nouvelles; centralisation toujours plus poussée, qui renforce l'unité à l'intérieur des ordres eux-mêmes et accentue leur dépendance à l'égard des congrégations romaines; ferveur plus grande et retour au respect des règles anciennes ${ }^{3} \ldots$ Pour bien étudier l'Église canadienne, il faut donc bien connâ̂tre, au point de départ, l'histoire de l'Église universelle.

3 R. Aubert, Le Pontificat de Pie IX, Paris, Bloud \& Gay, 1952, p. 456. 
Il faudrait analyser le plus sérieusement possible - en allant au fond des choses - l'apport des communautés françaises au milieu canadien. Je reprends ici des idées de Pierre Savard: "Ces com. munautés possèdent le dynamisme de la Restauration catholique qui caractérise la France au début du XIX ${ }^{e}$ siècle (...) Les Oblats, les Jésuites, les Clercs de Saint-Viateur et les Pères de Sainte-Croix apportent une mentalité qui va modifier le cours de l'histoire religieuse québécoise. Leur ultramontanisme ardent alimente des luttes politicoreligieuses naissantes. Leurs conceptions politiques réactionnaires consolident des tendances québecoises déjà existantes. (...) Tous ces religieux contribuent vivement à implanter ici la spiritualité du XIX siècle ${ }^{4}$.

Enfin, je ne vous apprends rien en vous disant qu'il y a beaucoup à faire en histoire de l'Église catholique du Canada. C'est pourquoi les forces ne doivent pas se disperser. Au contraire, il faut une collaboration de plus en plus étroite entre les chercheurs de diverses disciplines, mais aussi entre les historiens et les archivistes par exemple. 11 ne faut pas que l'on rende trop difficile l'accès de certains fonds; on devrait aller jusqu'à signaler à l'Institut d'histoire certains dossiers qui pourraient servir de base à des monographies. Nous avons à l'Institut des étudiants sérieux qui ne demandent pas mieux que de se pencher sur les phénomènes religieux. Tout cela permettra d'en arriver bientôt à la publication d'un grand nombre d'études d'histoire de l'Église canadienne et, nous l'espérons, à une vaste synthèse qui corrigera la constatation que je vous rappelais au début : «L'histoire du clergé canadien-français... reste à écrire. " C'est le meilleur vœu que nous puissions faire à la fin de cette journée consacrée à l'étude de quelques communautés religieuses installées au Québec.

Nive VoIsine

Institut d'histoire, Université Laval.

4 P. Savard, loc. cit., p. 265. 